\title{
Novel universal SARS-CoV DNA vaccine inducing neutralizing antibodies to huCoV-19/WH01, Beta, Delta and Omicron variants and T cells to Bat-CoV
}

\section{K. Sofia Appelberg}

Public Health Agency of Sweden

\section{Gustaf Ahlen}

Karolinska Institute

Negin Nikoyan

Karolinska Institutet

Jingy Yan

Karolinska Institutet

\section{Sofie Weber}

Adlego AB

Olivia Larsson

Adlego AB

Urban Höglund

Adlego AB

\section{Soo Aleman}

Karolinska Institutet

Friedemann Weber

Justus-Liebig University https://orcid.org/0000-0001-9737-337X

Emma Perlhamre

Karolinska University Hospital

Johanna Apro

Karolinska University Hospital

Eva-Karin Gidlund

Northx Biologics

Ola Tuvesson

Northx Biologics

Matteo Cadossi

IGEA SPa

SImona Salati

IGEA Spa

Hanna Tegel 
Royal Institute of Technology

\section{Sophia Hober}

Royal Institute of Technology

\section{Lars Frelin}

Karolinska Institutet https://orcid.org/0000-0001-7452-1667

ali mirazimi

Karolinska Institute

\section{Matti Sallberg ( $\nabla$ matti.sallberg@ki.se )}

Karolinska Institute https://orcid.org/0000-0002-8858-5132

\section{Letter}

\section{Keywords:}

Posted Date: January 20th, 2022

DOI: https://doi.org/10.21203/rs.3.rs-1276351/v1

License: (c) (1) This work is licensed under a Creative Commons Attribution 4.0 International License. Read Full License 
DRAFT for Nature Microbiology, January 19, 2022

Novel universal SARS-CoV DNA vaccine inducing neutralizing antibodies to huCoV-

19/WH01, Beta, Delta and Omicron variants and T cells to Bat-CoV

Sofia Appelberg ${ }^{1 \#}$, Gustaf Ahlén ${ }^{2 \#}$, Negin Nikouyann ${ }^{2 \S}$, Jingyi Yan ${ }^{2 \S}$, Sofie Weber ${ }^{3}$, Olivia Larsson $^{3}$, Urban Höglund ${ }^{3}$, Soo Aleman ${ }^{4}$, Friedemann Weber ${ }^{5}$, Emma Perlhamre ${ }^{6}$, Johanna Apro $^{6}$, Eva-Karin Gidlund ${ }^{7}$, Ola Tuvesson ${ }^{7}$, Simona Salati ${ }^{8}$, Matteo Cadossi $^{8}$, Hanna Tegel ${ }^{9}$, Sophia Hober ${ }^{9}$, Lars Frelin², Ali Mirazimi ${ }^{1,2 \ltimes}$, and Matti Sallberg ${ }^{2 \ltimes}$

1) Public Health Agency of Sweden, Solna, Sweden

2) Department of Laboratory Medicine, Karolinska Institutet, Sweden

3) Adlego AB, Uppsala, Sweden

4) Department of Infectious Disease, Karolinska University Hospital, and Department of Medicine Huddinge, Karolinska Institutet, Sweden

5) Institute for Virology, FB10-Veterinary Medicine, Justus-Liebing University Giessen,

\section{Germany}

6) Karolinska Trial Alliance, Karolinska University Hospital, Sweden

7) NorthX Biologics, Matfors, Sweden

8) IGEA Spa, Carpi, Italy

9) Department of Protein Science, KTH Royal Institute of Technology, Stockholm,

Sweden

$\#, \S, \not$ These authors contributed equally to this work 
DRAFT for Nature Microbiology, January 19, 2022

Corresponding author

Matti Sällberg, DDS, PhD, Professor

Karolinska Institutet,

Department of Laboratory Medicine,

Division of Clinical Microbiology, ANA Futura,

Alfred Nobels allé 8 ,

S-141 52 Huddinge

Phone: +46706082101

Email: matti.sallberg@ki.se 
DRAFT for Nature Microbiology, January 19, 2022

\section{INTRODUCTORY PARAGRAPH (148 words)}

The SARS-CoV-2 pandemic is constantly changing with new variants appearing that are more contagious (Alpha and Delta), evade the neutralising antibody (NAb) response (Beta), or both (Omicron). This is a challenge for vaccine development. We generated a novel universal SARSCoV-2 DNA vaccine containing the receptor binding domain (RBD) loops from the original huCoV-19/WH01, the Alpha, and the Beta variants, combined with the membrane and nucleoproteins from the huCoV-19/WH01 strain. This vaccine induced high levels of spike antibodies that crossreacted between the huCoV-19/WH01, Beta, and Delta spike proteins, and neutralized the huCoV-19/WH01, Beta, Delta and Omicron virus in vitro. The vaccine induced $\mathrm{T}$ cells to all vaccine proteins in mice and rabbits that recognized Bat-CoV $\mathrm{N}$ sequences. Finally, the vaccine protected K18 mice against lethal SARS-CoV-2 Beta variant infection, whereas only priming $\mathrm{N}$-specific $\mathrm{T}$ cells was $60 \%$ protective. This universal SARS-CoV vaccine candidate induces a uniquely broad functional immunity.

\section{MAIN TEXT (1428 words)}

The SARS-CoV-2 pandemic has completely altered the way the society handles new viral infections, with lockdowns of cities or even entire countries ${ }^{1}$. The extremely rapid development of vaccines by the scientific community and the pharmaceutical industry is an extraordinary achievement ${ }^{2}$. A previous SARS-CoV-2 infection or a vaccination with either adeno- or mRNA-based COVID-19 vaccines induces neutralizing antibodies (NAbs; ${ }^{3}$ ) and is effective in preventing symptomatic infection, and highly effective in preventing hospitalization or death ${ }^{4}$. However, the ability of the RNA genome of SARS-CoV-2 to undergo mutations and recombination poses continuous challenges ${ }^{5}$. It has recently been shown that among the new mutant strains, especially the newly emerged variants of concern (VOC), 
DRAFT for Nature Microbiology, January 19, 2022

anything from a few to multiple mutations in the receptor-binding domain (RBD) may render the current vaccines less effective against less severe breakthrough infections ${ }^{6,7}$. Several studies have highlighted that the Beta (B1.351), Delta (B.1.617.2), and Omicron (B.1.1.529) VOC may cause mild to moderate COVID-19 infections in those vaccinated ${ }^{8,9,10,11}$. Recent data suggest that a previous infection with the original huCoV-19/WH01 wildtype strain or the Alpha (B.1.117) variant induces NAbs that retain strong cross-reactivity with the Delta (B.1.617.2) variant, whereas infections with the Beta (B.1.351) and Omicron (B.1.1.529) can escape these NAbs ${ }^{12}$. Thus, the appearance of mild to moderate breakthrough infections are not surprising. Fortunately, greater changes would most likely be needed to completely avoid the host immunity. Mutations in variants that escape NAbs appear in surface-exposed epitopic regions of the virus that are recognized more, or less, uniformly across humans. In contrast, the high diversity of the human leucocyte antigen class I and II makes it much less likely, or maybe even impossible, for T cell-escape variants to appear in viruses such as SARS-CoV-2 that are only causing acute infections ${ }^{13,14}$. Consistent with this, it has been found that vaccine mediated protection against severe disease from variants such as the Delta (B.1.617.2) variant is maintained, despite a reduced protection against mild and moderate disease ${ }^{11}$. Consequently, although spike- and receptor binding domain (RBD)-specific B cells may lack cross-reactivity, the spike-specific T cells are still cross-reactive towards the different variants 13,14. This is further supported by the observation that T cells from SARS-infected patients that were infected with SARS-CoV in 2003 remain for 17 years and ar to a large extent crossreacting with SARS-CoV- ${ }^{15}$. Overall, this strongly supports the notion that T cells may be able to confer a broader cross-reactivity than NAbs both between SARS-CoV-2 mutants and between different SARS viruses. Thus new vaccines that combine sequences from the spike 
DRAFT for Nature Microbiology, January 19, 2022

protein from multiple variants, combined with highly conserved viral protein sequences may overcome these problems.

It is clear that bats are reservoir for the origin of human coronaviruses ${ }^{18}$. The spike proteins of SARS-CoV and SARS-CoV-2 induce cross-reactive T cells but poorly cross-reactive NAbs ${ }^{15}$. Among the structural proteins of SARS-CoV and SARS-CoV-2 the envelope protein $\mathrm{M}$ and the nucleocapsid protein $\mathrm{N}$ have a higher genetic similarity to other animal SARS-CoV viruses than the receptor-binding $S$ envelope protein ${ }^{18,19}$. T cells to these two antigens show a higher cross-reactivity across betacoronaviruses ${ }^{15}$. To take advantage of this, we designed a universal SARS-CoV (OC-2.4) vaccine containing receptor binding domain (RBD) loops of the S protein corresponding to the huCoV-19/WH01, Alpha and Beta variants, combined with the $\mathrm{M}$ and $\mathrm{N}$ proteins of the huCoV-19/WH01 variant ${ }^{20}$. An autoproteolytic P2A sequence was inserted between the RBD and the $\mathrm{M}$ and $\mathrm{N}$ proteins to avoid interference with the folding of the RBD. Figure 1a illustrates the design and the concept of inducing both NAbs and broadly cross-reactive T cells. As control vaccines we used either a full length S gene (huCoV-19/WH01) in pVAX1, pVAX plasmid without insert, and a recombinant S protein (huCoV-19/WH01) mixed with QS21 adjuvant. The aim of a universal vaccine is to induce broadly reactive antibodies and T cells. As the S protein differs significantly between SARS-CoV strains and variants, crossreactive T cells may be of a growing importance.

We first immunized Balb/c mice and found that they developed high levels of antibodies that bound recombinant S proteins of the huCoV-19/WH01, Beta and Delta variants (Figure 1b, d, $\mathrm{f}$, and $\mathrm{h}$ ) as well as to the $\mathrm{N}$ protein (Supplementary Figure 1). The S-specific antibodies effectively neutralized both the huCoV-19/WH01 and Beta variants of SARS-CoV-2 in vitro (Figure $1 \mathrm{c}$ and e). In addition, the mice developed T cells reactive to all components of the vaccine (depending on the mouse strain; data not shown). Importantly, the T cells to the $\mathrm{N}$ 
DRAFT for Nature Microbiology, January 19, 2022

protein were cross-reactive to sequences corresponding to the Bat-CoV N (Supplementary Figure 2).

Next, groups of mice and rabbits were immunized with the universal SARS-CoV DNA vaccine OC-2.4 and were analysed for anti-S levels, the ability to neutralize the Delta and Omicron variants in vitro, as well as the cross reactivity of $\mathrm{T}$ cells to the Bat $\mathrm{CoV} \mathrm{N}$ sequences (Supplementary Figures 2 and 3). In mice, priming with recombinant huCoV-19/WH01 S protein in adjuvant and boosting with the universal SARS-CoV DNA vaccine OC-2.4 effectively enhanced anti-S levels by 10-100-fold to huCoV-19/WH01 S protein (Figure 1f). Importantly, heterologous boosting with the universal DNA vaccine OC-2.4 induced higher neutralisation levels than homologous boosting (Figure 1g). Also, homologous boosting with the universal DNA vaccine OC-2.4 seemed superior in inducing NAbs to the Omicron variant (Figure $1 \mathrm{~g}$ ). In female rabbits we found that three doses of the OC-2.4 DNA vaccine induced high levels of anti-S antibody (mean \pm SD of $20160 \pm 28710$ endpoint titers) that effectively neutralized both Delta and Omicron variants of SARS-CoV-2 (Figure 1i). Thus, the inclusion of three RBD loops was effective at inducing broadly neutralizing antibodies to the huCoV-19/WH01, Beta, Delta and Omicron variants. As a comparison, three doses of a full length S DNA, or a recombinant spike protein in adjuvant, both corresponding to the huCoV-19/WH01 variant, were generally less effective in priming NAbs to the Beta, Delta and Omicron variants (Figure 1).

Finally, we analysed the ability of the universal DNA vaccine OC-2.4 to induce protective immune responses against a lethal challenge with the SARS-CoV-2 Beta variant in human ACE2 transgenic K18 mice. Groups of mice were immunized three times, three weeks apart, and two weeks after last dose the mice were challenged with $1 \times 10^{5}$ pfu of the SARS-CoV-2 Beta variant intranasally (Figure 2a), and then followed closely for symptoms and weight changes for thirteen days. The universal DNA vaccine OC-2.4 fully protected these mice against lethal 
DRAFT for Nature Microbiology, January 19, 2022

infection and showed complete protection against viral replication in the upper airways and in the spleen (Figure $2 b$ and c). The viral replication in the lungs was also significantly reduced as compared to controls (Figure 2c). In contrast, vaccination with recombinant S in QS21 adjuvant also protected against lethal infection, and gave low infection in the lungs, but viral replication could still be detected in the upper airways and in the spleen (Figure 2c). Interestingly, vaccination with the $\mathrm{N}$ protein in adjuvant, that only activates antibodies and $\mathrm{T}$ helper cell responses to $\mathrm{N}$, showed a $60 \%$ protection against lethal disease and viral replication in the upper airways, but less so in the lungs (Figure 2). This strongly support the notion that T cells alone have a key role against protection against severe disease ${ }^{33}$, and that the role may differ in the upper and the lower airways. This requires further studies. We found that the universal DNA vaccine effectively primes protective immune responses against severe disease and viral replication caused by a known immune escape variant of SARS-CoV-2.

In conclusion, with a virus like SARS-CoV-2 that shows an impressive ability to mutate and to spread also among a population with high vaccine coverage, new vaccine designs are needed. Here, we describe a unique universal SARS-CoV DNA vaccine that induces more broadly neutralizing activity against the huCoV-19/WH01, Beta, Delta and Omicron variants than standard spike-based vaccines. Our data supports clinical development of this completely new approach to vaccines against SARS-CoV-2 to complement existing vaccines, and potentially also protect against future SARS-CoV viruses that have replaced, or greatly changed the spike protein of SARS-CoV-2.

\section{ACKNOWLEDGEMENTS}

Our work was funded and supported by the OPENCORONA consortium that has received funding from the European Union's Horizon 2020 research and innovation programme under 
DRAFT for Nature Microbiology, January 19, 2022

grant agreement no. 101003666 . The study was also supported by The Swedish Research Council, The Swedish Cancer Society, Vinnova project CAMP (Contract no. 2017-02130), Knut and Alice Wallenberg foundation, Science for Life Laboratory (SciLifeLab), Erling-Persson family foundation, Karolinska Institutet (to LF), and by private donations to MS and AM.

\section{Figure Legends}

Figure 1. Schematic representation of the universal SARS-CoV-2 DNA vaccine OC-2.4 gene design has been given (a). Ability of the S-DNA and SARS-CoV-2 DNA vaccine OC-2.4 to induce antibodies against S protein of the huCoV-19/WH01 (b) and Beta variants (d), and the ability to neutralize these viruses in vitro ( $c$ and e). Also shown is the ability of the universal SARSCoV-2 DNA vaccine OC-2.4 to prime and boost antibodies to S protein (huCoV-19/WH01) following priming with a recombinant $S$ protein in adjuvant ( $f$ ) and the ability of these antibodies to neutralize the Delta and Omicron variants in vitro (g). Finally, three doses of the universal SARS-CoV-2 DNA vaccine OC-2.4 induce antibodies that cross react with S proteins from the huCoV-19/WH01, Beta, and Delta variants in mice (h), and three doses of the same vaccine induces antibodies that neutralize both the Delta and Omicron variants in vitro (i).

Figure 2. Evaluation of different vaccine strategies in the K18-hACE2 mice transgenic for the human ACE2 receptor. The experimental design has been given in (a). Three doses of the respective vaccines fully or partially protected the mice against severe disease as determined by weight loss (b) and viral replication in the nose, lungs and the spleen (c). Values have been given as cycle times (CT), where lower values indicate a higher viral load. Comparisons in the graph are shown as solid lines that indicate a $p<0.01$ (Mann-Whitney), and a dotted line indicate a $p<0.05$ (Mann-Whitney). 


\section{REFERENCES}

1. Smith, T.P. et al. Temperature and population density influence SARS-CoV-2 transmission in the absence of nonpharmaceutical interventions. Proc Natl Acad Sci U S A 118 (2021).

2. Golob, J.L., Lugogo, N., Lauring, A.S. \& Lok, A.S. SARS-CoV-2 vaccines: a triumph of science and collaboration. JCI Insight 6 (2021).

3. Varnaite, R. et al. Expansion of SARS-CoV-2-Specific Antibody-Secreting Cells and Generation of Neutralizing Antibodies in Hospitalized COVID-19 Patients. J Immunol 205, 2437-2446 (2020).

4. Lopez Bernal, J. et al. Effectiveness of the Pfizer-BioNTech and Oxford-AstraZeneca vaccines on covid-19 related symptoms, hospital admissions, and mortality in older adults in England: test negative case-control study. BMJ 373, n1088 (2021).

5. Miller, D. et al. Full genome viral sequences inform patterns of SARS-CoV-2 spread into and within Israel. Nat Commun 11, 5518 (2020).

6. Wall, E.C. et al. Neutralising antibody activity against SARS-CoV-2 VOCs B.1.617.2 and B.1.351 by BNT162b2 vaccination. Lancet 397, 2331-2333 (2021).

7. Kustin, T. et al. Evidence for increased breakthrough rates of SARS-CoV-2 variants of concern in BNT162b2-mRNA-vaccinated individuals. Nat Med (2021).

8. Madhi, S.A. et al. Efficacy of the ChAdOx1 nCoV-19 Covid-19 Vaccine against the B.1.351 Variant. N Engl J Med 384, 1885-1898 (2021).

9. Shinde, V. et al. Efficacy of NVX-CoV2373 Covid-19 Vaccine against the B.1.351 Variant. N Engl J Med 384, 1899-1909 (2021).

10. Sadoff, J. et al. Safety and Efficacy of Single-Dose Ad26.COV2.S Vaccine against Covid19. N Engl J Med 384, 2187-2201 (2021).

11. Sheikh, A. et al. SARS-CoV-2 Delta VOC in Scotland: demographics, risk of hospital admission, and vaccine effectiveness. Lancet (2021).

12. Liu, C. et al. Reduced neutralization of SARS-CoV-2 B.1.617 by vaccine and convalescent serum. Cell (2021).

13. Alter, G. et al. Immunogenicity of Ad26.COV2.S vaccine against SARS-CoV-2 variants in humans. Nature (2021).

14. Tarke, A. et al. Negligible impact of SARS-CoV-2 variants on CD4 (+) and CD8 (+) T cell reactivity in COVID-19 exposed donors and vaccinees. bioRxiv (2021). 
15. Le Bert, N. et al. SARS-CoV-2-specific T cell immunity in cases of COVID-19 and SARS, and uninfected controls. Nature 584, 457-462 (2020).

16. Maravelia, P. et al. Blocking Entry of Hepatitis B and D Viruses to Hepatocytes as a Novel Immunotherapy for Treating Chronic Infections. J Infect Dis 223, 128-138 (2021).

17. Hawman, D.W. et al. A DNA-based vaccine protects against Crimean-Congo haemorrhagic fever virus disease in a Cynomolgus macaque model. Nat Microbiol 6, 187-195 (2021).

18. Hu, B., Ge, X., Wang, L.F. \& Shi, Z. Bat origin of human coronaviruses. Virol J 12, 221 (2015).

19. Latinne, A. et al. Origin and cross-species transmission of bat coronaviruses in China. bioRxiv (2020).

20. Dai, L. et al. A Universal Design of Betacoronavirus Vaccines against COVID-19, MERS, and SARS. Cell 182, 722-733 e711 (2020).

21. Ahlen, G. et al. The SARS-CoV-2 N Protein Is a Good Component in a Vaccine. J Virol 94 (2020).

22. Shi, J. et al. Susceptibility of ferrets, cats, dogs, and other domesticated animals to SARS-coronavirus 2. Science 368, 1016-1020 (2020).

23. Shaan Lakshmanappa, Y. et al. SARS-CoV-2 induces robust germinal center CD4 T follicular helper cell responses in rhesus macaques. Nat Commun 12, 541 (2021).

24. Chandrashekar, A. et al. SARS-CoV-2 infection protects against rechallenge in rhesus macaques. Science 369, 812-817 (2020).

25. Feng, L. et al. An adenovirus-vectored COVID-19 vaccine confers protection from SARSCOV-2 challenge in rhesus macaques. Nat Commun 11, 4207 (2020).

26. Guebre-Xabier, M. et al. NVX-CoV2373 vaccine protects cynomolgus macaque upper and lower airways against SARS-CoV-2 challenge. Vaccine 38, 7892-7896 (2020).

27. Mercado, N.B. et al. Single-shot Ad26 vaccine protects against SARS-CoV-2 in rhesus macaques. Nature 586, 583-588 (2020).

28. van Doremalen, N. et al. ChAdOx1 nCoV-19 vaccine prevents SARS-CoV-2 pneumonia in rhesus macaques. Nature 586, 578-582 (2020).

29. Vogel, A.B. et al. BNT162b vaccines protect rhesus macaques from SARS-CoV-2. Nature (2021). 
DRAFT for Nature Microbiology, January 19, 2022

30. $\mathrm{Yu}, \mathrm{J}$. et al. DNA vaccine protection against SARS-CoV-2 in rhesus macaques. Science 369, 806-811 (2020).

31. Wrapp D, et al. Cryo-EM structure of the 2019-nCoV spike in the prefusion conformation. Science, 367, 1260-1263 (2020).

32. Hober S, et al. Systematic evaluation of SARS-CoV-2 antigens enables a highly specific and sensitive multiplex serological COVID-19 assay. Clin Transl Immunol, 10, 1-16 (2021).

33. Gao, Y. et al., Ancestral SARS-CoV-2-specific T cells cross-recognize the Omicron variant. Nature Medicine, https://doi.org/10.1038/d41591-022-00017-z 
DRAFT for Nature Microbiology, January 19, 2022

\section{METHODS}

\section{Animals}

Female BALB/c (H-2 $\left.{ }^{d}\right)$ mice were obtained from Charles River Laboratories, Sulzfeld, Germany. Female B6.Cg- $\mathrm{Tg}(\mathrm{K} 18-\mathrm{ACE} 2) 2 \mathrm{Pr} \mathrm{Imn} / \mathrm{J}$ mice (JAX stock \#034860) were purchased from Jackson Laboratory, USA. All mice were 8-12 weeks old at the start of the experiments and maintained under standard conditions at Preclinical laboratory (PKL), Karolinska University Hospital Huddinge, Sweden or Astrid Fagraeus Laboratory (AFL), Karolinska Institute, Solna, Sweden. Nine New Zealand White rabbits were purchased from Charles River, France and kept at AFL.

All animal procedures were granted by regional animal ethics committees (approvals Dnr. 03634-2020, 17114-2020 and 16676-2020.

\section{DNA Plasmids and recombinant proteins}

Vaccine candidate genes were generated based on the sequence from the huCoV-19/WH01 strain (Figure 1a). The genes contained the full Spike protein or a combination of the RBD, $N$ and $\mathrm{M}$ proteins, with a autoproteolytic $\mathrm{P} 2 \mathrm{~A}$ sequence. All sequences were codon optimized for expression in human cells and were synthesized by a commercial vendor (Genescript). Plasmids were grown in TOP10 Escherichia coli cells (Life Technologies) and purified for in vivo injections using Qiagen Endofree DNA purification kit (Qiagen) according to the manufacturer's instructions. The correct gene size was confirmed by restriction enzyme digests using BamHI and Xbal (Fast Digest; Thermo Fisher Scientific), and sequencing. Recombinant N protein was designed in-house and produced by Genescript (32878912). Recombinant $S, R B D$, and $M$ was purchased from Genescript. The spike proteins were produced as full length by transient protein production in mammalian cells (Expi293). To 
DRAFT for Nature Microbiology, January 19, 2022

facilitate trimerization of the full-length spike, a C-terminal T4 fibritin trimerization motif was included according to Wrapp et al ${ }^{31}$. Further a strep-tag, fused to the C-terminal was used for purification ${ }^{32}$. The beta version (B1.351) of the spike was produced with three mutations in the RBD part (K417N E484K N501Y).

\section{Peptides}

A total of 42 20-mer peptides with 10 aa overlap, corresponding to the huCoV-19/WH01 RBD (25 peptides), M (22 peptides) and N (41 peptides) and Bat N (42 peptides) were purchased from Sigma-Aldrich (St. Louis, MO). The peptides were divided in pools of 4-5 or 8-10 peptides/pool depending on experimental setup.

\section{Immunization and infection schedules in mice and rabbits.}

$B A L B / c\left(H-2^{d}\right)(n=5)$ mice were immunized up to three times with three weeks intervals, and sacrificed 2 weeks after the second immunization for spleen and blood collection. Twenty K18ACE2 mice were divided into 4 groups $(n=5)$ and immunized with indicated vaccines. Each K18hACE mouse received three immunizations with three weeks between each injection. Two weeks after the last immunization, the K18-hACE2 mice were infected with SARS-CoV-2 Beta. Immunization method in brief, BALB/c or K18-hACE2 mice (5 per group) were immunized intramuscularly in the Tibialis cranialis anterior muscle with $50 \mu \mathrm{g}$ plasmid DNA in a volume of $50 \mu \mathrm{L}$ sterile phosphate-buffered saline (PBS) by regular needle (27G) injection followed by in vivo electroporation using a Cliniporator2 device (IGEA) using 2 needle electrodes. Prior to vaccine injections, mice were given analgesic and kept under isoflurane anesthesia during the vaccinations. During in vivo electroporation (in both mice and rabbits ) a $1-\mathrm{ms} 600-\mathrm{V} / \mathrm{cm}$ pulse followed by a $400-\mathrm{ms} 60-\mathrm{V} / \mathrm{cm}$ pulse pattern was used to facilitate better uptake of the DNA. 
DRAFT for Nature Microbiology, January 19, 2022

In addition, groups of mice were injected subcutaneously at the base of the tail with recombinant SARS-CoV-2 spike (S) or nucleo (N) protein mixed (1:1) with QS21 adjuvant (GMP grade, Alpha diagnostics).

Each New Zealand White rabbits were immunized with $840 \mu \mathrm{g}$ OC-2.4 DNA vaccine or only formulation buffer (Tris-EDTA, $\mathrm{pH}$ 7.4). Injection were administered in the right Tibialis anterior muscle in $500 \mu \mathrm{L}$ followed by in vivo electroporation under anaesthesia using the GeneDriVe (IGEA) device and GeneGun electrode (IGEA) with a 4 needle electrode array at a depth of $21 \mathrm{~mm}$.

\section{Mice challenge model}

Two weeks post the last immunization the K18-hACE2 mice were challenged with $1 \times 10^{5} \mathrm{pfu}$ SARS-CoV-2 Beta via intranasal administration in a volume of $40 \mu$ l. The health of the animals was assessed daily for up to 13 days and evaluated based on several parameters, including body weight, general condition, piloerection, as well as movement and posture. At the time of euthanization, blood, nasal lavage, lungs and spleen was collected.

\section{Detection of IgG specific antibodies}

Serum from mice and rabbits were used for detection of immunoglobulins against $\mathrm{S}$ or $\mathrm{N}$ protein. In brief, plates were coated with $1 \mu \mathrm{g} / \mathrm{ml}$ recombinant S or N protein (Genescript) in $50 \mathrm{mM}$ Sodium Carbonate buffer $\mathrm{pH} 9.6$ overnight at $4{ }^{\circ} \mathrm{C}$. Plates were blocked by incubation with dilution buffer (phosphate-buffered saline, $2 \%$ goat serum, $1 \% \mathrm{BSA}$ ) for 1 hour at $37^{\circ} \mathrm{C}$. Serum was added in serial dilutions with a starting dilution of 1:60 and then in serial dilution 
DRAFT for Nature Microbiology, January 19, 2022

of 1:6. Serum antibodies were detected by an alkaline phosphatase conjugated goat antimouse IgG (Sigma A1047) 1:1 000 or goat anti-rabbit IgG (Sigma A2556) 1:1 000 and visualized using p-nitrophenyl phosphate substrate solution. Optical density (OD) was read at $405 \mathrm{~nm}$ with a $620 \mathrm{~nm}$ background. Antibody titers were determined as endpoint serum dilutions at which the OD value was at least three times the OD of the negative control (nonimmunized or control animal serum) at the same dilution.

\section{Detection of specific IFN- $\gamma$ producing T cells and antibodies}

Two weeks post last vaccination, splenocytes from each group of wildtype mice or peripherial blood mononuclear cells (PBMCs) were harvested and tested for their ability to induce specific T cells based on IFN- $\gamma$ secretion after peptide or protein stimulation for $48 \mathrm{~h}$ essentially as described ${ }^{16,17}$ using a commercially available enzyme-linked immunospot (ELISpot) assay (Mabtech).

\section{Virus propagation}

The SARS-CoV-2 huCoV-19/WH01, Beta, and Omicron strains were isolated from patient samples at the Public Health Agency of Sweden and confirmed by sequencing. The SARS-CoV2 Delta variant was provided by Dr. Charlotta Polacek Strandh, Statens Serum Institute, Copenhagen, Denmark. All variants were propagated on Vero-E6 cells and titered using a plaque assay as previously described ${ }^{3}$, with fixation after $72 \mathrm{hrs}$. The huCoV-19/WH01, Delta and Omicron strains used in this study was passaged 3 times and the Beta strain 2 times. 
DRAFT for Nature Microbiology, January 19, 2022

\section{Neutralization of SARS-CoV-2 in vitro}

Titer of neutralizing antibodies in serum from mice and rabbits were determined by CPE based microneutralization assay. For mice, serum from each vaccination group was pooled, while for rabbits serum from each individual was tested. Briefly, serum was heat inactivated at $56^{\circ} \mathrm{C}$ for 30 min before serial diluted 2-fold. Each dilution was conducted in quadruplets and mixed with 500 pfu of SARS-CoV-2 huCoV-19/WH01, Beta, Delta or Omicron in a 1:1 dilution. After 1 hour of incubation at $37^{\circ} \mathrm{C}, 5 \% \mathrm{CO}_{2} 100 \mathrm{uL}$ of serum-virus mix was added to Vero E6 cells on a 96-well plate $\left(20 \times 10^{4}\right.$ cells/well) and incubated for $72 \mathrm{hrs}$ at $37^{\circ} \mathrm{C}, 5 \% \mathrm{CO}_{2}$. CPE for each well was determined using a Nicon Eclipse TE300 microscope. As controls, wells with medium only, diluted serum only, virus only and serum known to contain SARS-CoV-2 neutralizing antibodies mixed with virus was included in each experiment.

\section{PCR/Viral RNA}

Trizol (Sigma-Aldrich) in a ratio of 1:3 was used to inactivate potential virus in nasal lavage samples $(50 \mu \mathrm{L})$ from SARS-CoV-2 infected K18-hACE2 mice. For lung and spleen, PBS was added to each sample $(1 \mathrm{~g} / \mathrm{ml})$ and pestles were used to crush the organs. Thereafter, the samples were centrifuged ( $5 \mathrm{~min}$ at $7000 \mathrm{rpm}$ ) and $50 \mu \mathrm{L}$ of each lung or spleen sample was added to Trizol (1:3). Total RNA was extracted using the Direct-zol RNA Miniprep kit (Zymo Research) according to the manufacturer's instructions. Viral RNA were thereafter measured by quantitative real-time polymerase chain reaction (qRT-PCR) using TagMan Fast Virus 1-Step master mix (Thermo Fisher Scientific) with primers and probe for the SARS-CoV-2 E gene.

Forward: 5' - ACAGGTACGTTAATAGTTAATAGCGT-3'

Reverese: 5' - ATATTGCAGCAGTACGCACACA -3'

Probe: FAM- ACACTA GCC ATC CTT ACT GCG CTT CG MGB 
DRAFT for Nature Microbiology, January 19, 2022

For lung and spleen samples, mouse ACTB mix (Thermo Fisher Scientific) was used as endogenous control. The PCR reaction was performed using a capillary Roche LightCycler 2.0 system.

\section{Statistical analysis}

Data was analyzed with use of on GraphPad Prism V.5 software and Microsoft Excel V.16.13.1. 

a)
IgE-leader RBD (253 aa)
RBD (253 aa)
\begin{tabular}{l|l} 
RBD $(253$ aa) & P2A \\
\hline
\end{tabular}
M(216 ав)
N (419 aa)

huCoV/WHO1

B.1.117

B1.351

huCoV/WH01 strain
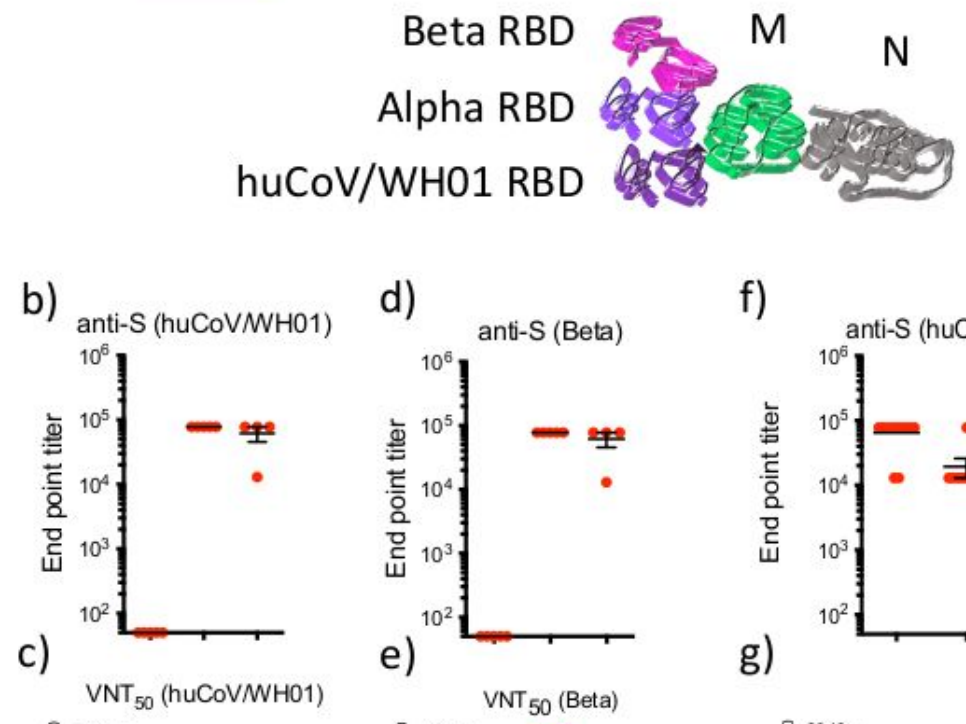

f) anti-S (huCoV/WH01)
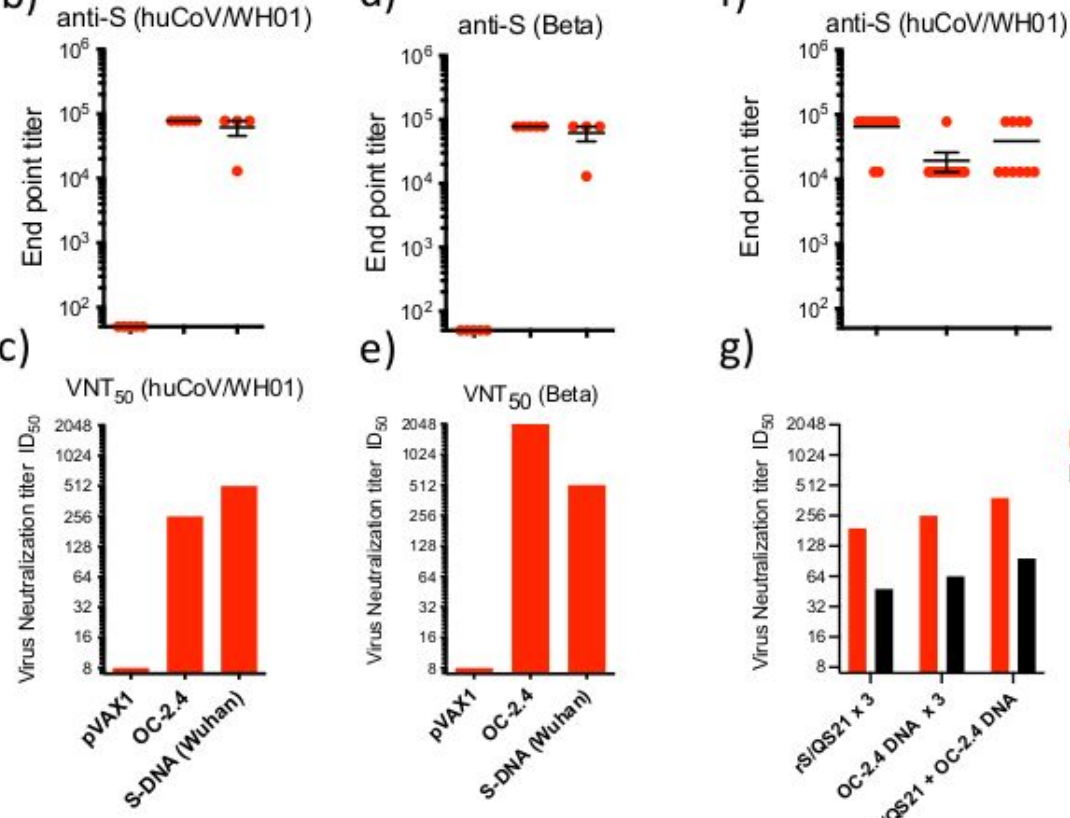

g)

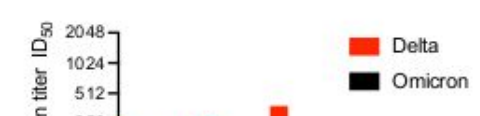

Vaccine

h)

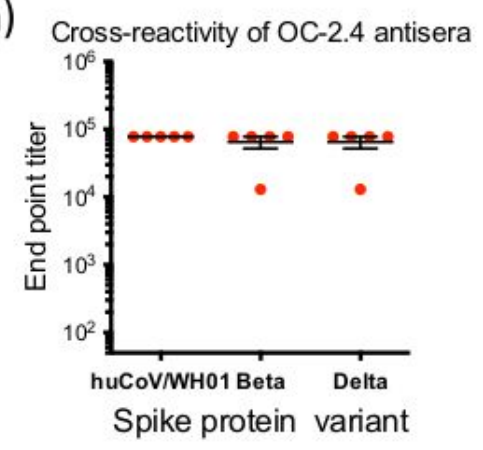

i)

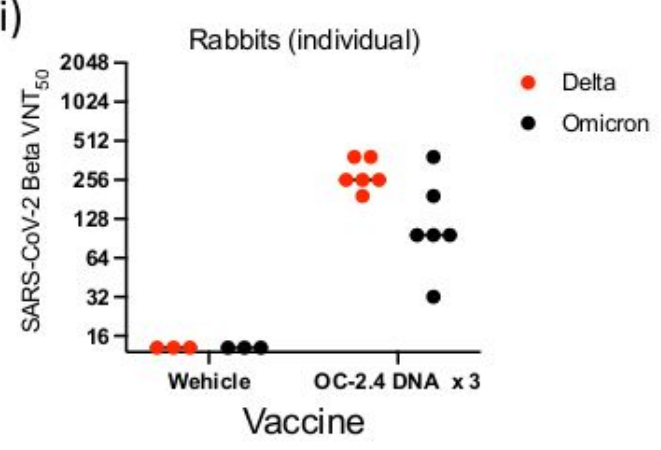

Figure 1

\section{Figure 1}

Schematic representation of the universal SARS-CoV-2 DNA vaccine OC-2.4 gene design has been given (a). Ability of the S-DNA and SARS-CoV-2 DNA vaccine OC-2.4 to induce antibodies against S protein of the huCoV-19/WH01 (b) and Beta variants (d), and the ability to neutralize these viruses in vitro (c and e). 
Also shown is the ability of the universal SARSCoV-2 DNA vaccine OC-2.4 to prime and boost antibodies to $S$ protein (huCoV-19/WH01) following priming with a recombinant $S$ protein in adjuvant (f) and the ability of these antibodies to neutralize the Delta and Omicron variants in vitro (g). Finally, three doses of the universal SARS-CoV-2 DNA vaccine OC-2.4 induce antibodies that cross react with S proteins from the huCoV-19/WH01, Beta, and Delta variants in mice (h), and three doses of the same vaccine induces antibodies that neutralize both the Delta and Omicron variants in vitro (i).

a)

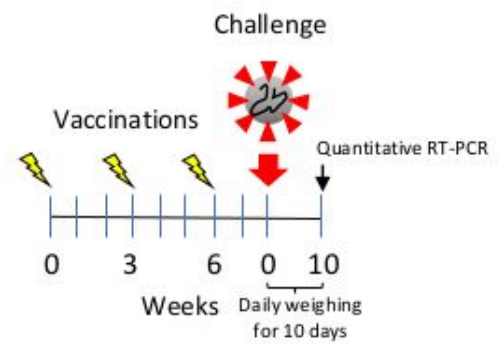

b)
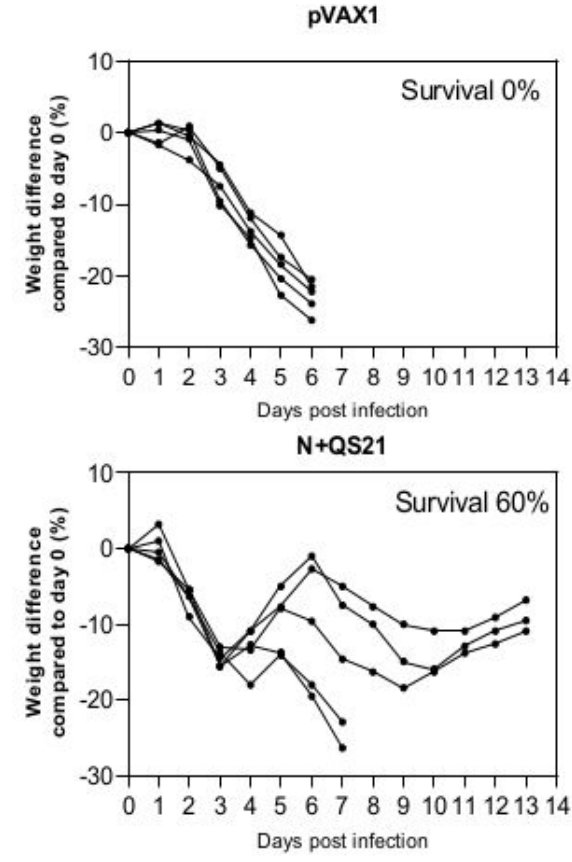
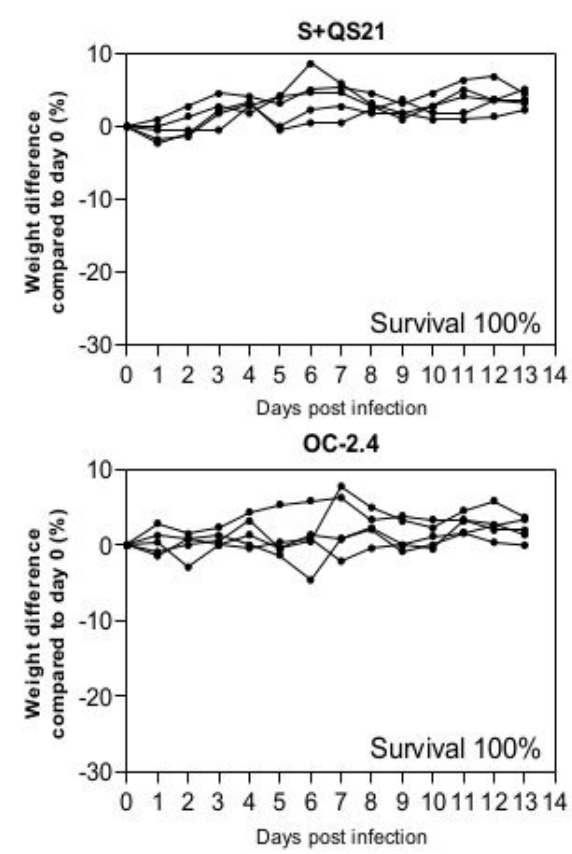

c)

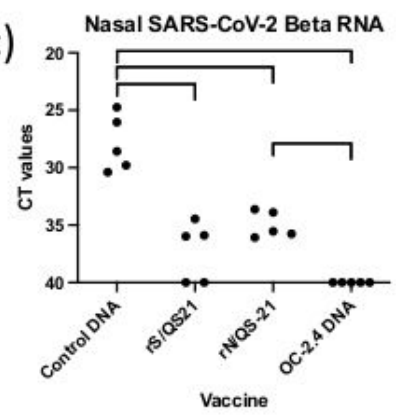

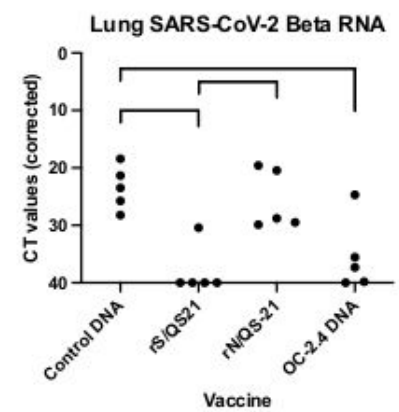

Vaccine

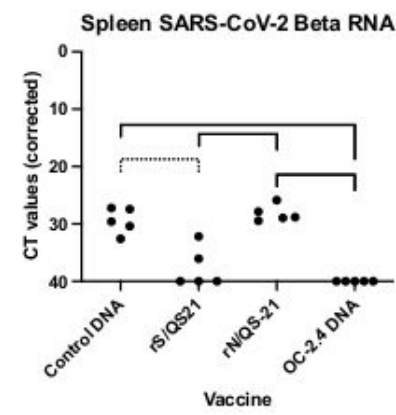

Figure 2 


\section{Figure 2}

Evaluation of different vaccine strategies in the K18-hACE2 mice transgenic for the human ACE2 receptor. The experimental design has been given in (a). Three doses of the respective vaccines fully or partially protected the mice against severe disease as determined by weight loss (b) and viral replication in the nose, lungs and the spleen (c). Values have been given as cycle times (CT), where lower values indicate a higher viral load. Comparisons in the graph are shown as solid lines that indicate a $p<0.01$ (MannWhitney), and a dotted line indicate a $\mathrm{p}<0.05$ (Mann-Whitney).

\section{Supplementary Files}

This is a list of supplementary files associated with this preprint. Click to download.

- suppfig3.jpg

- suppfig2.jpg

- suppfig1.jpg 